\title{
Treating Periodontitis with the Use of Essential Oil and Herbs
}

\author{
Kopiga Ananthathavam, Jaiganesh Ramamurthy
}

\begin{abstract}
Oral diseases are major health problems among which dental caries and periodontal diseases are the most important preventable diseases. When these conditions are not arrested,they cause formation of periodontal pocketsPeriodontal diseases havebeen linked to bacteria like Actinobacillus actinomycetemcomitans, Treponema denticola, T.socranskii, P.intermedia and Porphyromonas gingivalis ${ }^{[1]}$

The need to develop an alternative prevention and treatment option to antibacterial agents, is to avoid sideeffects of the antibiotics and the increased resistance of the bacteria. Hence, natural herbs and ayurvedic drugs have become a subject of importance ${ }^{[2]}$
\end{abstract}

KEYWORDS: Aloe-vera,bacteria,herbs,periodontal disease,

\section{INTRODUCTION}

Chronic low grade infections like gingivitis are thought to contribute to heart disease. Diseases that affect one or more of the periodontal tissue like alveolar bone, periodontal ligament, cementum and gingiva are known as periodontitis. These periodontal diseases occur when bacteria in plaque infect the gums and the bones that support the teeth. Both Actinobacillus actinomycetemcomitans and Porphyromonas ginigivalis, along with multiple deep pockets in the gum are thought to cause aggressive periodontal disease. P.ginigvalis produces enzymes like arginine-specific cysteine proteinase that disrupts the immune system and leads to periodontal connective tissue disruption. Periodontitis is of two types, namely : Chronic periodontitis and aggressive periodontitis. Chronic periodontitis is the most common form whereas aggressive periodontitis occurs in population below 30 years of age. Non-surgical therapy is useful in mild to moderate periodontitis whereas surgical therapy is useful in advanced cases. Local drug delivery systems like mouth rinses, irrigating solutions and sustained release devices are used for the targeted delivery of antimicrobial agents like fibers, strips and compacts, films, microparticles, gels and nanoparticles. This review article summarizes the effects of natural products like Acacia catechu willd, Aloe vera, Chamomile, Azadirachta indica, Glycyrrhiza glabra for the treatment of periodontal diseases ${ }^{[3]}$ and also the effects of Centella asiacata and Punica granantum. ${ }^{[4]}$

\section{HERBS FOR THE MANAGEMENT OF PERIODONTAL DISEASE :}

2.1 Acacia catechu willd: (fam:Fabaceae and subfamily:Mimosoideae)

Acacia catechu willd (AC) is widely used in the treatment of skin diseases. Ac is not only useful in the treatment of diarrhea, dysentery, colitis and gastric cancer but also in the treatment of mouth, gum and throat diseases like gingivitis and stomatitis. AC and Katha (a concentrated filtered extract) is cooling, digestive and an astringent. The decoction of bark mixed with milk cures cold and cough. The extracts of AC have anti-pyretic, anti-inflammatory, anti-diarrhoeal, hypoglycemic, hepatoprotective, antioxidant and antimicrobial activities. Melancholia, conjunctivitis, haemoptysis, cattarah, cough, pruritis, leprosy, leukoderma, skin diseases, helminthiasis, anorexia, dysentery, diarrhea, foul ulcers and wounds, fever, anaemia, diabetes and pharyngodynia can be treated with the heart-wood of AC. This heart -wood contains catechin, catechutannic acid, epicatechin, catechin tetramer, dicatechin, gallocatechin, kaempferol, taxifolin, isorhamnetin, L-arabinose, D-galactose, D-rhamnose, (+)Afzelechinn and aldobiuronic acid. ${ }^{[5]}$ Catechin is used as a haemostatic and Taxifolin has anti-bacterial, anti-fungal, anti-inflammatory and antioxidant properties.

Uses of AC in the management of periodontal disease: A composition of AC powder(91\%), Menthol( $2.7 \%)$ and Camphor $(6.3 \%)$ removes plaque,stain or patches and cleans and polishes tooth surface without an abrasive action. The powder of AC is used for cleaning and polishing tooth surface without an abrasibe action whereas powder of menthol and camphor is used as a flavouring agent. A clinical study on this dentifrice tooth powder reported $87-95 \%, 70-72 \%$ and $80-95 \%$ reductions in plaque, gingivitis and dental calculus respectively, in about 15 days of treatment ${ }^{[6]}$ 


\subsection{Aloe vera (Aloe barbadensis miller)}

It belongs to the family Asphodelaceae (Liliaceae). It is a shrubby, perennial, xerophytic, pea-green colour plant. It grows mainly in the dry regions. In India, it is found in Rajasthan, Andhra Pradesh, Gujarat, Maharashtra and Tamil Nadu. Aloe vera consists of vitamins, enzymes, minerals, sugars, lignin, saponins, salicylic acids and amino acids. ${ }^{[7]}$

\section{Effects of Aloe vera:}

- It has good wound healing activity.

- It has a protective effect against radiation damage to the skin.

- It inhibits the cyclooxygenase pathway and decreases prostaglandin E2 formation from arachidonic acid. Hence, it is a potent anti-inflammatory agent.

- It is a potent laxative.

- It has anti acne and antiseptic effect.

- Aloe vera is used in the treatment of Seborrheic dermatitis, psoriasis vulgaris, skin burns, type 2 diabetes, genital herpes, HIV infection, cancer prevevntion, ulcerative colitis, mucolsitis, radiation dermatitis, lichen planus, acne vulgaris, aphthous stomatitis, frostbite.

- Streptococcus pyogenes and Strptococcus faecalis are two microorganisms inhibited by aloe vera. ${ }^{[8]}$

- Heggers et al suggested that Aloe vera in vivo inhibits bacteria that causes inflammation and thereby enhances wound healing.

Processed Aloe vera preparation inhibited the growth of Candida albicans.

\section{Uses of Aloe vera in the management of periodontal diseases:}

A composition of olive oil extract, black walnut green hulls, clove leaf, thyme herb, grapefruit seed extract, chamomile flower, green tea leaf, Oregano leaf, peppermint oil, Aloe vera, Calendula flower, Echinacea purpura, gota kola extract, Cinnamon bark, Eucalyptus leaf, Lavender oil, etc was effective in preventing and treating oral diseases and maintaining good oral health. This composition provided a permanent solution for oral disorders like gingivitis, periodontal disease, stomatitis and halitosis. Build-up of tartar on teeth is prevented and it suppresses oral and throat cancer. The composition maybe in the form of a solution that is diluted before use or in the form of powder that is added to beverages, candies, toothpaste, dental chew, whitening molds, etc.

\subsection{Chamomile: (Family:Asteraceae/Compositae)}

Chamomile is recognized in the Western culture. Due to its calming and spasmolytic properties, it is a common ingredient in the herbal teas and also is an ingredient in health and beauty products for its soothing and anti-inflammatory effects on skin. Two species of Chamomile are Matricaria chamomilla (German chamomile) and Chamaemelum nobile (Roman chamomile). German chamomile is most commonly used. $50-65 \%$ of the total volatile oil content contain (-)-alpha-bisabolol and chamazulene. Other components of the oil include (-)alpha-bisabolol oxide A and B, spiroethers, sesquiterpenes, cadinene, farnesene, furfural, spathulenol and proazulene.Chamomile oil (concentration of $25 \mathrm{mg} / \mathrm{mL}$ ) demonstrates antibacterial activity against Grampositive bacteria such as Bacillus subtilis, Staphylococcus aureus, Strptococcus mutans and Strptococcus salivarius. It also has fungicidal activity against Candidia albicans. ${ }^{[9]}$ Alpha-bisabolol provides the antibacterial, antifungal, anti-inflammatory, and anti-ulcer activity. ${ }^{[10]}$ Chamomile helps the body to resist or destroy microorganisms. Azulene is bactericidal to staphylococcus and Streptococcus infections.

Uses of Chamomile in the management of Periodontal diseases: ${ }^{[11]}$

Chamomile helps to reduce inflammation from periodontitis and also reduces the level of unhealthy bacteria in the mouth. In order to expose the gum to this herb, Chamomile tea is taken or mouthrinses and toothpastes containing Chamomile is taken to overcome periodontal infections.

\subsection{Azadirachta indica (Neem)}

Azadirachta indica is well known in India. More than 135 components have been isolated from neem. The main ingredients are nimbin, nimbinin and nimbidin. The neem leaves contain 6-desacetylnimbinene, nimbandiol, nimbolide and quercetin, $\mathrm{n}$-hexacosanol and nonacosane. Beta-sitosterol is present in all parts of the plant $^{[12]}$

- Neem oil along with the bark and leaf extracts have been therapeutically used to control leprosy, intestinal helminthiasis, respiratory disorders, constipation and as a health promoter.

- Rheumatism, chronic syphilitic sores and indolent ulcers have also been treated by neem.

- Bark, leaf, root, flower and fruit cure blood morbidity, biliary afflictions, itching, skin ulcers, burning sensation and pthysis. ${ }^{[13]}$

- Neem leaf extracts have anti-arrythmic, anti-arthritic, anti-viral, anti-diabetic and antioxidant. 
- It is hepato-protective.

- It has anti-ulcer, anti-malarial, anti-fungal and anti-carcinogenic activity. ${ }^{[14]}$

- In vitro, it inhibits Vibrio cholera, Klebsiella pneumonia, M.tuberculosis and M.pyogenes. ${ }^{[15]}$

\section{Uses of Neem leaf extract in the management of Periodontal diseases:}

People residing in the villages of India use Neem twigs to brush their teeth in order keep their gum free of disease and infection. A study shows that Neem leaf extract is used to treat dental plaque and gingivitis.

${ }^{[16]}$ Micro-organisms found in inflamed gum are resistant to tetracycline and penicillin but not to the Neem leaf extracts. Also Neem leaf extracts cause no allergic reaction in the gingiva. ${ }^{[17]}$ Advanced gum disease characterized by an inflammation of the gums and the membranes covering the roots of the teeth is known as Pyorrhoea. When this condition was treated with neem-based toothpaste and mouthwash, the bleeding gums healed, the secretion from pockets around the teeth ceased and the blue-tinted gums returned to healthy pale pink colour. ${ }^{[18]}$ The herbal formulation comprising of active fractions from Azadirachta indica, Citrullus colocynthis and Cucumis sativus is useful for preventing dental plaque and ginigivitis in humans and is also used as an antimicrobial agent for preventing disease.

\subsection{Glycyrrhiza glabra (Liquorice root)}

Glycyrrhiza glabra is also known as liquorice and sweet wood. It is native to the Mediterranean and certain areas of Asia. Licorice and Liquorice possess sweet taste and has extensive pharmacyological effects.

- Liquorice is used in the treatment of upper respiratory ailments like cough, hoarseness, sore throat and bronchitis. ${ }^{[19]}$

- In Japan, Liquorice extracts have been used for more than 60 years to treat chronic hepatitis.

- It is also thought to have a therapeutic benefit against human immunodeficiency virus, cytomegalo virus and herpes simplex virus.

- In the treatment of ulcers, Deglycyrrhizinated liquorice (DGL) is used.

- Topical liquorice preparations have been used to soothe and heal skin eruptions like psoriasis and herpertic lesions.

- It is used in digestive disorders.

- It possess good anti-bacterial, anti-fungal, antioxidant, antitussive, hepatoprotective and anti-inflammatory activity.

\section{Uses of liquorice root in the management of Periodontal diseases: ${ }^{[20]}$}

Moon et al described the use of plant extract powder in oral formulations for the prevention and treatment of periodontal diseases and tooth decay. The plant extract was loaded into a porous powder carrier that was coated with a water insoluble coating material. The extract contained Pine, Liquorice, Cassia seed, Cinnamon, Nothosmyrnium root, Sophora, lonicera flower, Platycodon, green tea, day flower, Korean angelica root, liriope rhizome, moutan, Arabian myrrh, seseleos radix, Angelicae Dahuricae Radix, Lagersromemia indica, morusk, ginger, Sanguinaria, Asaram, Cimicifuga, Chinese galls, Grapefruit seed, Lycium root, Cnidium, Alpinia katsumadai Hayata, Gardenia, Lythrum salicaria L, dandelion, propolis, falvanoid, nepta herb, Reynoturia japonica Houtt, scutelleria, machilia, black adzuki bean, chamomile, ratanhia or sage oil as single or in combination. The water insoluble coating easily disintegrated in the oral cavity and the active ingredients are released. The composition maybe formulated as a toothpaste, oral cleaner, oral purifier, etc. ${ }^{[21]} 18$-beta-glycyrrhetinic acid (GA) is an anti-inflammatory compound extracted from liquorice root extract. There was a dramatical reduction in the infection-induced bone loss in IL-10 deficient mice when GA was administered prophylactically or therapeutically.Initially GA was thought to exert its anti-inflammatory activity through down regulation of 11-beta hydroxysteroid dehydrogenase-2 (HSD2). HSD2 converts active glucocorticoid to inactive forms. But, GA did not reduce HSD2 expression in gingival tissue. GA inhibits periodontitis by inactivation of NF.

\subsection{Centella asiatica and Punica granatum (Pomegranate) ${ }^{[22]}$}

It is a small herb belonging to the family Apiaceae. It is used for internal and external applications of various diseases. The principle components include Asiatic acid, madecassic acid and asiaticoside. Extract of Cetella asiatica increased cellular proliferation and collagen synthesis at the wound site.

Punica granatum Linn belongs to the family Punicaceae. The pericarp of the fruit has hydrolysable tannins which inhibits prostaglandin synthetase. It possessed low acute toxicity and caused no skin irritation. Studies show that attachment levels improved in pockets that had initial depth of 5-8mm, when treated with Centella asiatica and Punica granatum. 


\section{CONCLUSION:}

The global need for an alternative prevention, treatment options and products for oral disease are safe, effective and economical arises due to the rise in disease incidence, increased resistance by pathogenic bacteria to currently used antibiotics. ${ }^{[23]}$

Not only teeth is important, but gum care is also very important. Therefore, in this review herbs like Acacia catechu willd, Aloe vera, Chamomile, Azadirchta indica, Glycyrrhiza gabra, Centella asiatica and Punica granatum are found to be useful in the prevention, treatment and maintenance of periodontal diseases.

\section{REFERENCES:}

[1] 2 and 3) Jai Ganesh and Lakshmi (2011). Unfolding gift of nature- Herbs for the management of Periodontal disease. Journal of Pharmacology research. 1:2576

[2] Grindwit Sastrvaha, Pusadee Yotnuengnit, Prakongsiri Booncong and preecha Sangtherapitikul. Adjunctive Periodontal treatment with Centella asiatica and Punica granatum extracts. 2:107

[3] Sharma P. C., Yelne M. B., Denni T. J., Database on Medicinal Plants used in Ayurveda, Volume 1, Central Council For Research in Ayurveda and Siddha, Dept.of ISM \& H, Min. of Health \& Family Welfare, Govt. of India, 216-224. 2000

[4] Jai Ganesh and Lakshmi(2011). The unfolding gift of nature- Herbs for the management of periodontal disease. Journal of Pharmacology research. 3:2577

[5] Shelton M. Aloe vera, its chemical and therapeutic properties. Int J Dermatol 1991;30:679-83.

[6] Atherton P. The essential Aloe vera: The actions and the evidence. 2nd ed. 1997.

[7] Chithra R Sajithlal GB, Chandrakasan G. Inß uence of aloe vera On collagen characteristics in healing dermal wounds in rats. Mol cell Biochem 1998;181:71-6.

[8] Azghani AO,Williams I, Holiday DB, Johnson AR. A beta-linked mannan inhibits adherence of Pseudomonas aeruginosa to human lung epithelial cells. Glycobiology 1995;5:39-44.

[9] Romanowski, R.: US20050158252A1 (2005)

[10] Garnick JJ, Singh B,Winkley G. Effectiveness of a medicament containing silicon dioxide, aloe, and allantoin on aphthous ulcers. Oral Surg Oral Med Oral Pathol Oral Radiol Endod 1998;86:550-556.

[11] Berry M. The chamomiles. Pharm J 1995;254:191-193.

[12] Cinco M, Banfi E, Tubaro A, 3. et al. A microbiological survey on the activity of a hydroalcoholic extract of chamomile. Int $\mathrm{J}$ Drug Res 1983;21:145-151.

[13] Aggag ME, Yousef RT. Study of antimicrobial activity of chamomile oil. Planta

[14] Med 1972;22:140-144.

[15] Szelenyi I, Isaac O, Thiemer K. Pharmacological experiments with compounds of chamomile. III. Experimental studies of the ulcerprotective effect of chamomile Planta Med 1979;35:218-227.

[16] Isaac O. Pharmacological investigations with compounds of chamomile i. on the pharmacology of (-)-alpha-bisabolol and bisabolol oxides (review) Planta Med 1979; 35:118-124.

[17] Isaac O, Thiemer K. Biochemical studies on chamomile components/III. In vitro studies about the antipeptic activity of (-)-alpha-bisabolol Arzneimittelforschung 1975; 25:1352-1354.

[18] Chopra, R. N., Nayer, S. L. and Chopra, I. C., Glossary of Indian Medicinal Plants, CSIR, New Delhi, 1956.

[19] Chevalier, Andrew. The Encyclopedia of Medicinal Plants. DK Publishing Inc.,1996

[20] Food Plants of the World: An illustrated guide by Ben-Erik van Wyk,Timber Press Publishers, October 2005; Page no: 1-15

[21] Siddiqui, S., Curr. Sci., 1942, 11, 278-279

[22] Mitra, C. R., Garg, H. S. and Pandey, G. N., Phytochemistry,1971, 10, 857-864

[23] Balasenthil, S., Arivazhagan, S., Ramachandran, C. R. and Nagini, S., J .Ethnopharmacol ., 1999, 67, 189-195

[24] Almas, K., Indian J. Dent. Res., 1999, 10, 23-26.

[25] www.neemamerica.com/PDF/Neem_Teeth-Jan2005.pdf

[26] http://www.neemamerica.com/research.asp

[27] http://www.neemamerica.com/research.asp

[28] Dirsch V, Faculty of Life Sciences, Universitat Wien, 2006.

[29] Setzer N, Natural Products Drug Discovery, 1999

[30] Moon, H.S., Lee, B.R., Lee, K.H.: US20036503541 (2003).

[31] Zhu, J.: EP1504763A4 (2006)

[32] 18beta-Glycyrrhetinic Acid Inhibits Periodontitis via Glucocorticoid-Indepen- dent NF-?B Inactivation in IL-10 Deficient Mice Hajime Sasaki Et al Journal of Periodontal Research Volume 45, Issue 6, pages 757-763, December 2010

[33] Moon, H.S., Lee, B.R., Lee, K.H.: US20036503541 (2003).

[34] Zhu, J.: EP1504763A4 (2006)

[35] 18beta-Glycyrrhetinic Acid Inhibits Periodontitis via Glucocorticoid-Indepen- dent NF-?B Inactivation in IL-10 Deficient Mice Hajime Sasaki Et al Journal of Periodontal Research Volume 45, Issue 6, pages 757-763, December 2010

[36] 21) Moon, H.S., Lee, B.R., Lee, K.H.: US20036503541 (2003).

[37] 22) Grindwit Sastrvaha, Pusadee Yotnuengnit, Prakongsiri Booncong and preecha Sangtherapitikul. Adjunctive Periodontal treatment with Centella asiatica and Punica granatum extracts. 2:107 